Research Article

\title{
Impact of Magnetohydrodynamics on Stagnation Point Slip Flow due to Nonlinearly Propagating Sheet with Nonuniform Thermal Reservoir
}

\author{
Zeeshan Khan ${ }^{D},{ }^{1}$ Haroon Ur Rasheed ${ }^{D},{ }^{2}$ Saeed Islam, ${ }^{2}$ Sahib Noor, ${ }^{1}$ Waris Khan, ${ }^{3}$ \\ Tariq Abbas, ${ }^{1}$ Ilyas Khan $\left(\mathbb{1},{ }^{4}\right.$ Seifedine Kadry $\left(\mathbb{1},{ }^{5}\right.$ Yunyoung Nam $\left(\mathbb{1},{ }^{6}\right.$ \\ and Kottakkaran Sooppy Nisar ${ }^{7}$ \\ ${ }^{1}$ Sarhad University of Science and Information Technology, Peshawar 25000, KPK, Pakistan \\ ${ }^{2}$ Department of Mathematics, Abdul Wali Khan University Mardan, Mardan 23000, KPK, Pakistan \\ ${ }^{3}$ Department of Mathematics, Kohat University of Science and Technology, Kohat 26000, KPK, Pakistan \\ ${ }^{4}$ Department of Mathematics, College of Science Al-Zulfi, Majmaah University, Al-Majmaah 11952, Saudi Arabia \\ ${ }^{5}$ Department of Mathematics and Computer Science, Faculty of Science, Beirut Arab University, Beirut, Lebanon \\ ${ }^{6}$ Department of Computer Science and Engineering, Soonchunhyang University, Asan 31538, Republic of Korea \\ ${ }^{7}$ Department of Mathematics, College of Arts and Sciences, Prince Sattam Bin Abdulaziz University, Wadi Al-Dawaser, \\ Al-Kharj 11991, Saudi Arabia
}

Correspondence should be addressed to Ilyas Khan; i.said@mu.edu.sa and Yunyoung Nam; ynam@sch.ac.kr

Received 5 August 2019; Revised 12 January 2020; Accepted 14 January 2020; Published 19 February 2020

Academic Editor: Fazal M. Mahomed

Copyright (C) 2020 Zeeshan Khan et al. This is an open access article distributed under the Creative Commons Attribution License, which permits unrestricted use, distribution, and reproduction in any medium, provided the original work is properly cited.

In this analysis, we introduced heat convective aspects of stagnation point movement of a magnetohydrodynamic (MHD) stream on a nonlinear oscillating plane with the impacts of velocity and heat slips with variable heat reservoir. By using some appropriate transformations, the governing differential equations are switched into an ordinary differential equation. The semianalytics technique called Homotpy Analysis Method (HAM) has been applied to evaluate the ordinary differential equations. For convergence achievement, a numerical method BVPh2-midpoint method is also applied and an outstanding agreement is found. The impacts of the governing constraints on flow, motion, and temperature distributions are investigated in detail. We observed that the temperature distribution increases with nonlinear heat reservoir parameter. Our results, in some limiting situations, matched well with previously published results, which approve that our obtained results are correct.

\section{Introduction}

It is, to some extent, understood that the present generation depends on the achievements of physical sciences which are based on production industries. Magnetohydrodynamics (MHD) boundary-layer flow on an elongating surface is important because of its frequent uses in industrial engineering and various production processes such as the aerodynamic squeezing of polymers, rolling at high temperature, cooling control technology, and glass fiber production. The magnetohydrodynamics can display certain characteristics in heat conductivity as it has both fluid as well as magnetic features. Raju et al. [1] investigated an extensive study of the least squares finite element method over a varying boundary layer explored the stagnation flow. Gorla [2] studied a viscoelastic (a nonclassical) liquid of stagnation movement in pulsating magnetic field. Gorla concluded that the shear stress coefficients are directly proportional to the magnetic field. Takhar et al. [3] investigated magnetohydrodynamic nonlinear stagnation point interface movement. Besser et al. [4] presented a technique of annihilating the magnetic field in the limit of MHD equations for a noncompressible ionized fluid having constant viscosity and resistivity as exchange parameters. Massoudi and Ramezan 
[5] examined the heat convective features of fluid (i.e., viscoelastic) at stagnation point flow. Ariel [6] studied multidirectional stagnation point movement of viscoelastic material. Mahapatra and Gupta [7] derived an exactly matched solution of Navier-Stokes equations which characterizes a uniform axisymmetric stagnation point flux towards an elongating surface. It is observed that the flow shows a boundary-layer arrangement when the velocity of the elongating sheet is smaller than the free stream velocity and a counter boundary layer is made when the starching sheet velocity is greater than the free stream velocity. Abel et al. [8] studied the influence of varying temperature source on magnetohydrodynamics heat convection in the fluid thin layer on a nonuniform elongating surface.

Yazdi et al. [9] evaluated the magnetohydrodynamics slip streaming on the nonuniform porous elongating sheet in the existence of a chemical process. The slip stream takes place if the distinctive size of flow regime is much smaller or the flow pressure is weak. Hsiao [10] has worked on an incompressible uniform MHD stagnation point movement of a second-order viscoelastic liquid and thermal conduction caused by a flow oscillating surface, and it is observed that viscoelastic liquid flow thermal effect is better than nonviscoelastic liquid flow thermal effect. Rasheed et al. [11] analyzed numerical and analytical investigation of thin-film nanofluid flow over an angular surface. Roşca et al. [12] presented an analysis for the uniform boundary-layer movement and heat transformation of a non-Newtonian fluid in the stagnation point along with unsteady propagating plane sheet of the unrestricted flow slip velocity and presented a dual (upper and lower branch) solution for certain variables. Dessie and Kishan [13] studied the magnetohydrodynamics over boundary-layer stream and heat transformation of fluid material with varying viscosity, permeable channel, heat reservoir, and viscous dissipation, respectively. Rasheed et al. [14] investigated the two-dimensional viscoelastic fluid with nonuniform heat generation over permeable stretching sheet with slip condition. Hassan [15] described the charge carrying viscous interfacelayer flow and thermal transfer. Shen et al. [16] studied the MHD-varied heat transfer flow near a stagnation point flow on a nonuniform stretching surface with velocity slip. Tufail and Ali [17] investigated the effects of fluid flow and heat convection due to a nonlinear stretching sheet. Gireesha et al. [18] examined the hydromagnetic heat conduction in a mixed viscous fluid over uniform stretching thermovariant surface and observed the effects of different relevant parameters on flow and heat transfer. Zaidi and Mohyud-Din [19] studied the convective transformation of heat and significance of MHD effects in different technologies.

Khan et al. [20-25] discussed the impact of various nonNewtonian fluid materials for wire coating analysis in the presence of magnetic field. Kuman et al. [26] investigated the characteristics of entropy generation in radiative flow of CNTs Casson nanofluid in rotating channels with heat source. Khan et al. [27] studied the analytical and numerical solutions of an Oldroyd 8-constant fluid in double-layer optical fiber coating as a coating material. Nandeppanavar et al. [28] studied heat conduction over a nonuniform elongating surface with unsteady heat reservoir and flexible temperature at boundary [28], and fluid layer flow is caused by a nonuniform elongating surface [29], magnetohydrodynamic stagnation point stream movement and heat convection [30], and seconddegree slip effects over flow movement [31]. Ibrahim and Ul Haq [32] studied the heat transformation in fluid and MHD stagnation point movement and observed that the thickness of heat conducting layer rises when the thermophoresis factor reduces with Prandtl number Pr.

By studying the foresaid investigations, it is found that there is no research on the stagnation point movement of a non-Newtonian fluid and thermal convection with thermal jump and momentum in the existence of an unsteady heat reservoir as a result of the moving sheet. In this analysis, we studied the said constraints on flow and heat transfer.

\section{Mathematical Formulation}

Figure 1 demonstrates physical configuration of the considered problem. Here, we measured two-dimensional steady, flow, and transfer of heat analysis in case of an incompressible fluid in the occurrence of transverse magnetic field strength $B(x)$ affected normally on flow moment which give us a unique form given as follows:

$$
B(x)=B_{0} x^{(n-1) / 2}, \quad B_{0} \neq 0,
$$

where $n$ is a constant and $x$ is a coordinate along the plate measured from the leading edge. The plate is moving inside or outside the origin with the velocity $u_{W}(x)=a x^{n}$ in an exterior (in viscid) flow of the velocity $u_{e}(x)=a x^{n}$, where $u$ and $v$ are the corresponding velocity components in the $x$ and $y$ directions, respectively. Here, $T_{W}(x)$ is assumed as a temperature of the plate and ambient fluid is $T_{\infty}$ which is the constant temperature. The governing equations of continuity, momentum, and energy equations are as follows:

$$
\begin{aligned}
\frac{\partial u}{\partial x}+\frac{\partial u}{\partial y} & =0, \\
u \frac{\partial u}{\partial x}+v \frac{\partial u}{\partial y} & =\mu_{e} \frac{\mathrm{d} \mu_{e}}{\mathrm{~d} x}+v \frac{\partial^{2} u}{\partial y^{2}}-\frac{\sigma B^{2}(x)}{\rho} u, \\
u \frac{\partial T}{\partial x}+v \frac{\partial T}{\partial y} & =\alpha \frac{\partial^{2} T}{\partial y^{2}}+\frac{q^{\prime \prime \prime}}{\rho c_{p}},
\end{aligned}
$$

where $\rho$ is the conductivity (electrical) of the fluid, $\alpha$ is the thermal diffusivity constant, and $u$ and $v$ are velocity components in the $x y$-plane, respectively.

The initial and boundary conditions are as follows:

$$
\begin{aligned}
& u=v, T=T_{\infty}, \quad \text { for any } x, y \\
& v=0, u=a x^{n}+N_{1} v \frac{\partial u}{\partial y}, T=T_{w}(x)+S_{1} \frac{\partial T}{\partial y} \\
& \text { at } y=0, u-\mu_{e}(x) a x^{n} \\
& T \longrightarrow T_{\infty}, \quad \text { as } y \longrightarrow \infty
\end{aligned}
$$




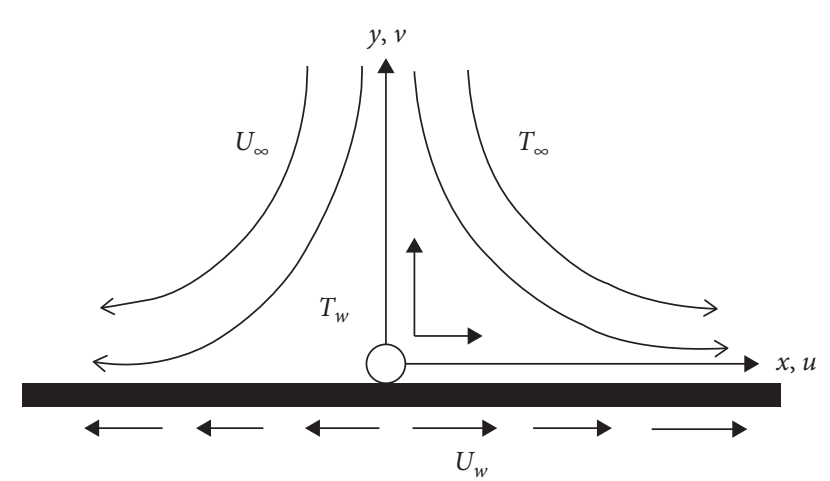

FIGURE 1: Geometrical sketch of the model problem.

We guess the velocity slip factor $N_{1}$ and the temperature slip factor $S_{1}$ change with $x$ in the form $N_{1}=N x^{((1-n) / 2)}$ and $S_{1}=S x^{((1-n) / 2)}$, respectively, where $N$ and $S$ are positive slip constants. Furthermore, it is expected that surface temperature $T_{W}(x)=T_{\infty}+T_{0} x^{P}$, where $T_{0}$ is the characteristics temperature parameter and $P$ is the wall temperature parameter. It is concluded from $N_{1}=N x^{((1-n) / 2)}$ and $S_{1}=S x^{((1-n) / 2)}$ against physical point of view, $n$ should vary in the range $0 \leq n \leq 1$. If $n>1$, then $N_{1}$ and $S_{1}$ become singular at $x$ chose to the leading edge of the plate. It is remembered that the boundary layer does not start at $x=0$ but starts in the vicinity of the leading edge of the plate [33].

Therefore, the solution for $n>1$ is realizable from the mathematical point of view. $C_{P}$ is the specific heat at constant pressure, $\sigma$ is the electrical conductivity, $B_{0}$ is the applied magnetic field, $\mu$ is the viscosity, $T$ is the temperature, $k$ is the thermal conductivity of the fluid, and $q^{\prime \prime \prime}$ is the rate of nonuniform heat generation/absorption coefficient and defined as

$$
q^{\prime \prime \prime}=\left(\frac{k u_{w}(x)}{x v}\right)\left[A^{*}\left(T_{w}-T_{\infty}\right) f^{\prime}+B^{*}\left(T-T_{\infty}\right)\right],
$$

where $A^{*}$ and $B^{*}$ are the conditions of space- and temperature-dependent heat generation/absorption, respectively. We notice that $A^{*}>0$ and $B^{*}>0$ for the internal thermal generation and $A^{*}<0$ and $B^{*}<0$ for the internal thermal absorption [28].

\section{Transformation of PDEs into ODEs}

Using self-similar solution by means of the similarity function $f$, define

$$
\begin{aligned}
& u=a x^{n} f^{\prime}(\eta), \\
& v=-\sqrt{\frac{a v(n+1)}{2}} x^{((n-1) / 2)}\left[f(\eta)+\frac{n-1}{n+1} \eta f^{\prime}(\eta)\right] .
\end{aligned}
$$

Evaluating equations (2)-(4) with the help of the boundary conditions given in (5) and using the similarity transformation techniques,

$$
\begin{aligned}
\psi & =\sqrt{\frac{2 a v}{n+1}} x^{((n+1) / 2)} f(\eta), \\
\theta(\eta) & =\frac{T-T_{\infty}}{T_{w}-T_{\infty}}, \\
\eta & =\sqrt{\frac{a(n+1)}{2}} x^{((n-1) / 2)} y,
\end{aligned}
$$

where $\psi$ denotes stream function, defined by $u=(\partial \psi / \partial y)$ and $v=-(\partial \psi / \partial x)$, and $v=(\mu / \rho)$ denotes kinematic viscosity. The governor partial differential equations (PDEs) given in equations (3) and (4) are transferred into ordinary differential equations. By using equations (6)-(8) in equations (2)-(4), we acquire a set of ordinary differential equations given below:

$$
\begin{array}{r}
f^{\prime \prime \prime}+f f^{\prime \prime}+\left(\frac{2 n}{n+1}\right)\left[1+\left(f^{\prime}\right)^{2}\right]-M_{n} f^{\prime}=0, \\
\frac{1}{\operatorname{Pr}} \theta^{\prime \prime}+f \theta^{\prime}-\left(\frac{2 p}{n+1}\right) f^{\prime} \theta+\left(\frac{2}{n+1}\right) \frac{1}{\operatorname{Pr}}\left(A^{*} f^{\prime}+B^{*} \theta\right)=0,
\end{array}
$$

where $\operatorname{Pr}=(v / \alpha)$ denotes the Prandtl number, and the transferred boundary conditions given in equation (5) takes the form

$$
\left.\begin{array}{l}
f(0)=0, f^{\prime}(0)=\lambda+\beta f^{\prime \prime}(0), f^{\prime}(\infty)=1 \\
\theta(0)=1+\sigma \theta^{\prime}(0), \theta(\infty)=0
\end{array}\right\},
$$

where $\lambda=(c / a)$ is the moving parameter with $\lambda>0$ corresponding to downstream movement of the plate from the origin, while $\lambda<0$ corresponding to the moving of the plate into the origin $\beta=N \sqrt{(a v(n+1) / 2 v)}$ is the velocity slip parameter and $\sigma=S \sqrt{(a(n+1) / 2 v)}$ denotes the temperature slip parameter. It is worth noticing when $n=1$ (stagnation point flow), $p=0$ (isothermal plate), and $\beta=\lambda=\sigma=0$, equations (9) and (10) along with the boundary conditions (11) become identical for $m=1$ (stagnation point flow and heat transfer) [34].

Physical quantities for local skin-friction coefficient $C_{f}$ and local Nusselt number $N u_{x}$ in this problem are defined as

$$
\begin{aligned}
C_{f} & =\frac{\tau_{w}}{\rho u_{e}^{2}(x)}, \\
N u_{x} & =\frac{x q_{w}}{k\left(T_{w}-T_{\infty}\right)},
\end{aligned}
$$

where skin friction (shear stress) along the plate is $\tau_{w}$, and wall heat $q_{w}$ is given by

$$
\begin{aligned}
& \tau_{w}=\mu\left(\frac{\partial u}{\partial y}\right), \\
& q_{w}=-k\left(\frac{\partial T}{\partial y}\right)_{y=0} .
\end{aligned}
$$

From equations (8), (12), and (13), we acquired 


$$
\begin{gathered}
\sqrt{\operatorname{Re}_{x}} C_{f}=\sqrt{\frac{n+1}{2}} f^{\prime \prime}(0), \\
\operatorname{Re}_{x}^{-(1 / 2)} N u_{x}=\sqrt{\frac{n+1}{2}}\left[-\theta^{\prime}(0)\right],
\end{gathered}
$$

where $\operatorname{Re}_{x}=\left(u_{e}(x) x / v\right)$ denotes the Reynolds number.

\section{Solution by HAM (Homotopy Analysis Method)}

The governing nonlinear partial differential equations (3) and (4) are converted into nonlinear ordinary differential equations (9) and (10). To find the solutions of equations (9) and (10), five boundary conditions are required: three on equation of motion and two on equations of temperature, respectively. But here $f^{\prime \prime}(\eta)$ and $\theta^{\prime}(\eta)$ are missing boundary conditions; hence, solving the boundary value problem of equations (9) and (10) is difficult. Therefore, in the boundary conditions given in equation (11) we replace infinity to the finite value. Equations (9) and (10) with boundary condition (11) are solved analytically by HAM and numerical by BVPh2-midpoint methods. The optimal HAM [35-37] gives better results compared with perturbation techniques and other conventional investigative techniques. Firstly, the optimal HAM gives us a remarkable flexibility to pick the equation type of linear subproblems. Secondly, the optimal HAM works regardless of the possibility that there does not exists any small/large physical parameter in determining equations and boundary/initial conditions. Particularly, unlike perturbation and other analytic techniques, the optimal HAM gives us an advantageous approach to guarantee the convergence of a series solution by presenting the supposed convergence control parameter into the series solution.

\section{Convergence of the Optimal HAM Method}

The auxiliary parameters $f$ and $\theta$ have a leading purpose of controlling the convergence of homotopic solutions. To get convergent solutions, we take the suggested values of these parameters. For this reason, residual errors are noticed for the momentum, and thermal energy equations by initiating the expressions are given as

$$
\begin{aligned}
& \Delta_{m}^{f}=\int_{0}^{1}\left[R_{m}^{f}\left(\xi, \hbar_{f}\right)\right]^{2} \mathrm{~d} \xi, \\
& \Delta_{m}^{\theta}=\int_{0}^{1}\left[R_{m}{ }^{f}\left(\xi, \hbar_{\theta}\right)\right]^{2} \mathrm{~d} \xi .
\end{aligned}
$$

The convergence of the parametric values computed through optimal HAM is listed in Table 1 using the values of the parameters $\beta=1.2, \lambda=0.5, \operatorname{Pr}=2.0, \phi=0.1$, and $\gamma=0.1$, while the error decay for 10 th-order approximation is shown in Figure 2.

Here, $\Delta_{m}^{t}=\Delta_{m}^{f}+\Delta_{m}^{\theta}$ denotes the total discrete square residual error which is used to obtained optimal convergence control parameters.
TABLE 1: The average residual square errors $\left(\Delta_{m}^{t}\right)$.

\begin{tabular}{lccc}
\hline Order & $h_{f}$ & $h_{\theta}$ & $\Delta_{m}^{t}$ \\
\hline 4 & 0.00375856 & 0.00491626 & 0.00019201 \\
8 & 0.0000381264 & 0.0000168988 & 0.0000168988 \\
12 & $4.3 \times 10^{-7}$ & $2.4 \times 10^{-7}$ & $1.0 \times 10^{-8}$ \\
16 & $5.1 \times 10^{-9}$ & $2.0 \times 10^{-10}$ & $2.2 \times 10^{-12}$ \\
20 & $6.2 \times 10^{-11}$ & $2.8 \times 10^{-12}$ & $2.6 \times 10^{-14}$ \\
24 & $7.7 \times 10^{-12}$ & $2.0 \times 10^{-14}$ & $0.5 \times 10^{-15}$ \\
28 & $9.7 \times 10^{-15}$ & $2.6 \times 10^{-17}$ & $3.12 \times 10^{-20}$ \\
30 & $1.1 \times 10^{-15}$ & $1.7 \times 10^{-18}$ & $1.21 \times 10^{-22}$ \\
\hline
\end{tabular}

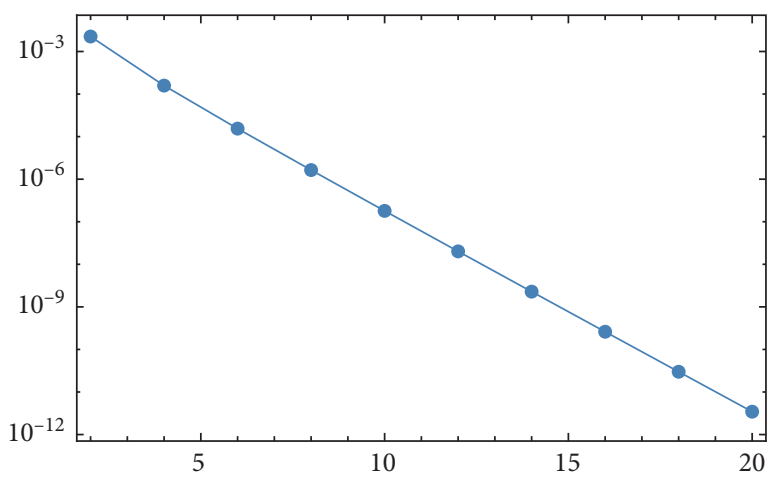

Figure 2: Error decay for the 10th-order approximation.

\section{Comparison of HAM/Numerical and Published Work}

Table 2 shows the comparison of HAM, BVPh2-midpoint method, and published work reported by Bejan [34], and good agreement has been found.

\section{Discussion on Results}

In this paper, an analysis is introduced to investigate magnetohydrodynamic slip flow and thermal convection of the stagnate point of an elongating surface along with nonuniform oscillating plane along a free flow. The momentum and heat convection equations for the nearest neighbor layer of interface have been explained analytically and derived for various analytical equations for temperature profile. The outcomes of the semianalytical computations are displayed in different figures. The characteristic parameters which are investigated in this paper are power-law index $n$, boundary-layer temperature $P$, Prandtl number Pr, magnetic field parameter $M$, and spatial dependent heat reservoir parameters $A^{*}$ and $B^{*}$, respectively. We now continue to discuss the results. Figures 3 and 4 demonstrate the stream velocity profiles for the various values of $\lambda$ (oscillating parameter) and $\beta$ (velocity slip parameter), respectively. It has been observed that the rising of $\lambda$ and $\beta$ causes reduction in the width of the boundary-layer movement.

Figure 5 describes velocity and flow profiles of various input of power index variable $n$ while the other parameters are kept fixed. It is observed that rising the power index variable, the stream movement and velocity rise, which cause an increment in the width of the momentum boundary wall layer. Figure 6 exhibits the velocity sketch for various input 
TABLE 2: The comparison of HAM, BVPh2, and published work.

\begin{tabular}{lccc}
\hline$\eta$ & Present work & BVPh2-midpoint method & Bejan [34] \\
\hline 0.0 & 0.1214461 & 0.1214463 & 0.1214465 \\
1.0 & 0.5183797 & 0.5183795 & 0.5183796 \\
2.0 & 0.6061305 & 0.6061309 & 0.6061304 \\
3.0 & 0.6230634 & 0.6230636 & 0.6230638 \\
4.0 & 0.6332310 & 0.6332313 & 0.6332312 \\
5.0 & 0.6499570 & 0.6499574 & 0.6499573 \\
6.0 & 0.6779332 & 0.6779336 & 0.6779335 \\
7.0 & 0.7214930 & 0.7214933 & 0.7214934 \\
8.0 & 0.7856251 & 0.7856255 & 0.7856252 \\
9.0 & 0.8761650 & 0.8761656 & 0.8761653 \\
10.0 & 1.0 & 1.0 & 1.0 \\
\hline
\end{tabular}

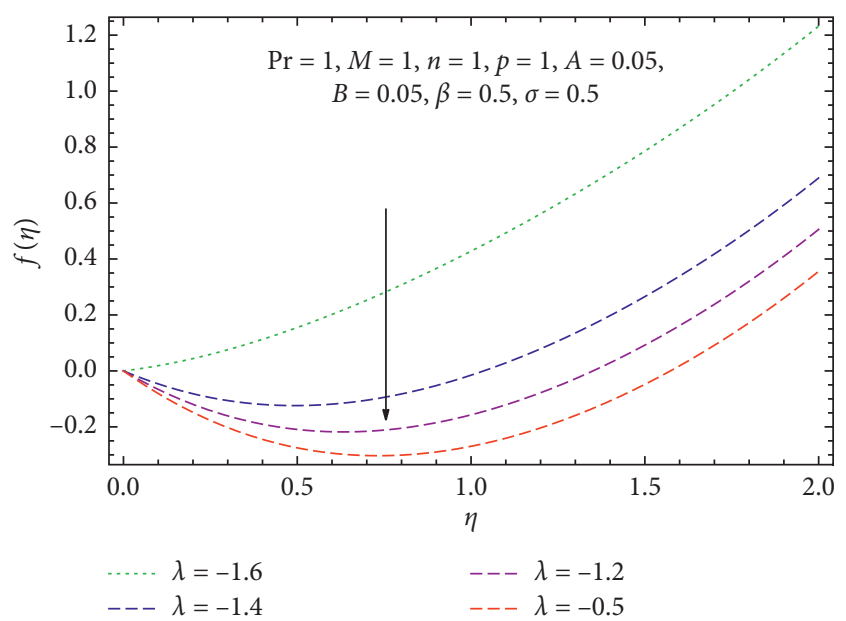

Figure 3: Flow profile for different values of $\lambda$.

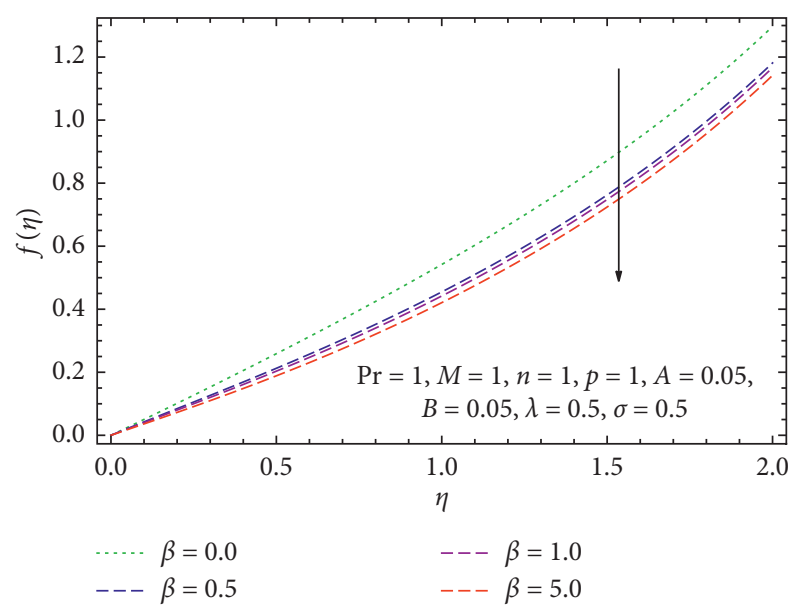

FIgURE 4: Flow profile for different values of $\beta$.

of $M$. From here, it is detected that velocity along with the boundary-layer thickness reduces when $M$ is increased. This may be due to the application of electromagnetic force (Lorentz force).

Figures 7 and 8 depict the velocity profiles for the various inputs of $\lambda$ and $\beta$. In these figures, we can see that as the value of $\lambda$ advances, the thickness of velocity increases but we

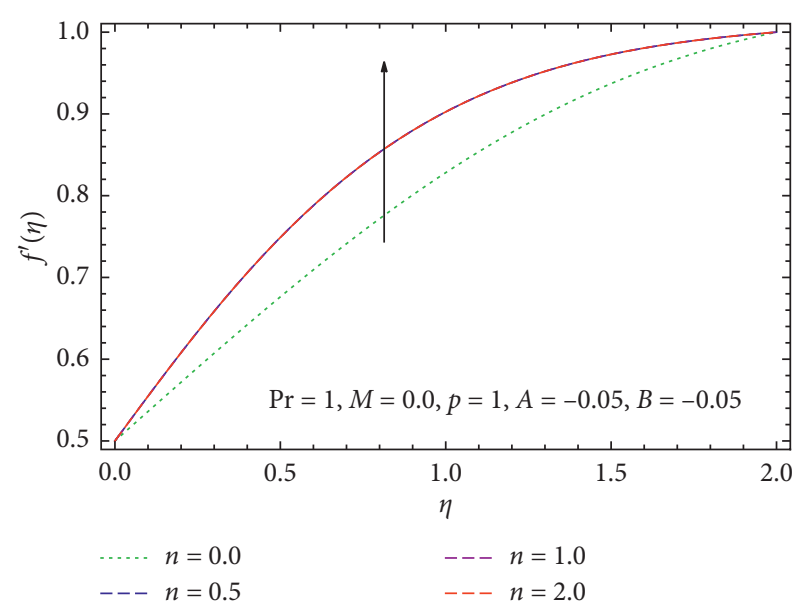

Figure 5: Velocity profile for different values of $n$ and different parameters.

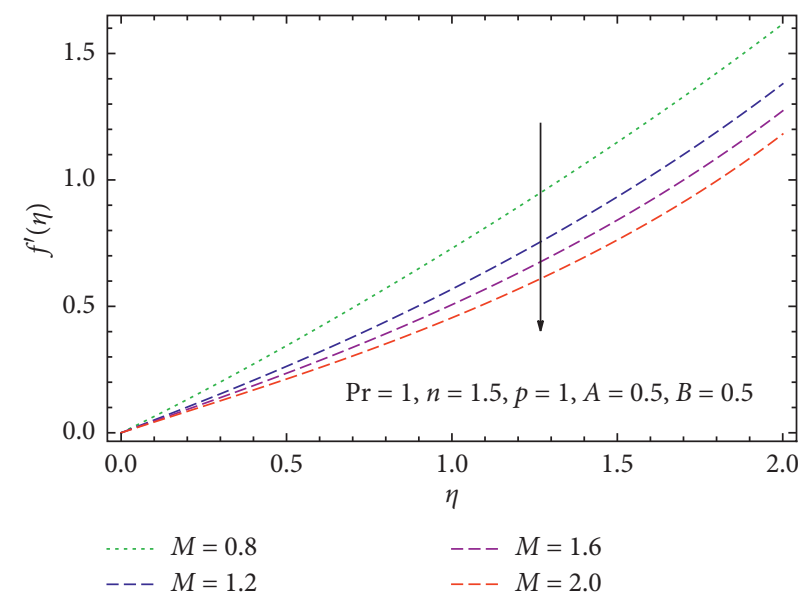

Figure 6: Velocity profile for different values of $M$.

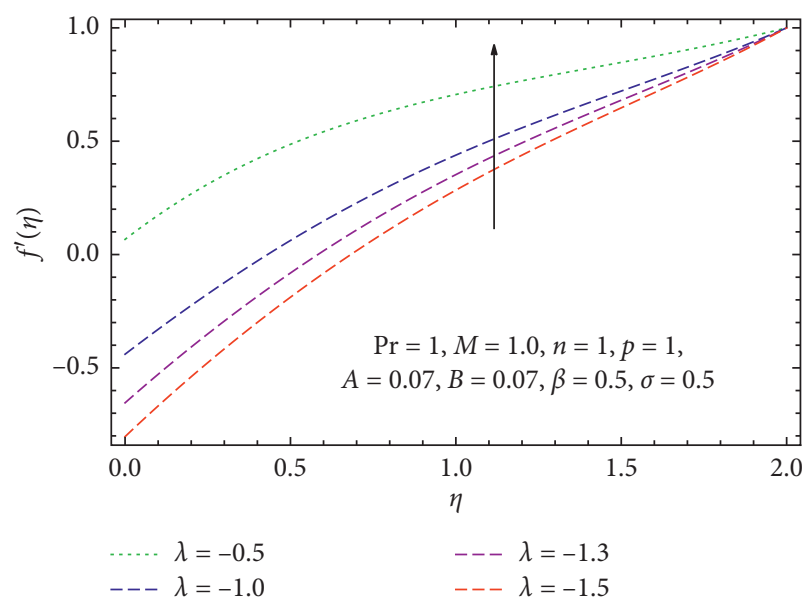

Figure 7: Velocity profile for different values of $\lambda$.

observed a reverse result when the velocity slip parameter $\beta$ increases. Figures 9 and 10 show the skin-friction profile for different values of $\lambda$ and $\beta$. In these profiles, it is clear that the 


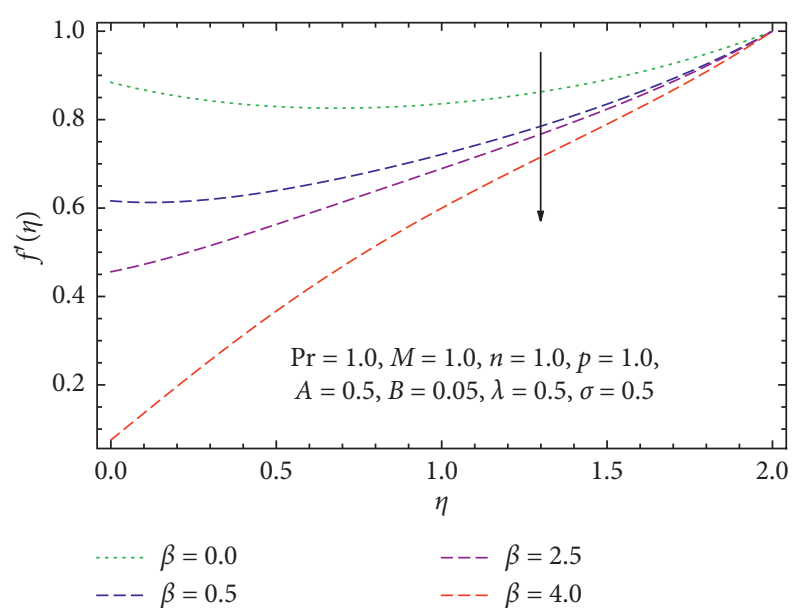

FIgURE 8: Velocity profile for different values of $\beta$.

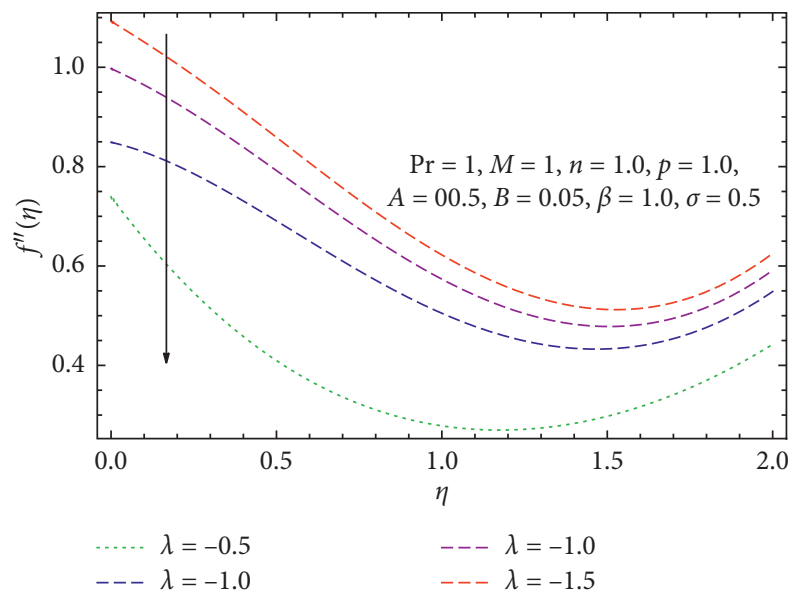

Figure 9: Skin-friction profile for different values of $\lambda$.

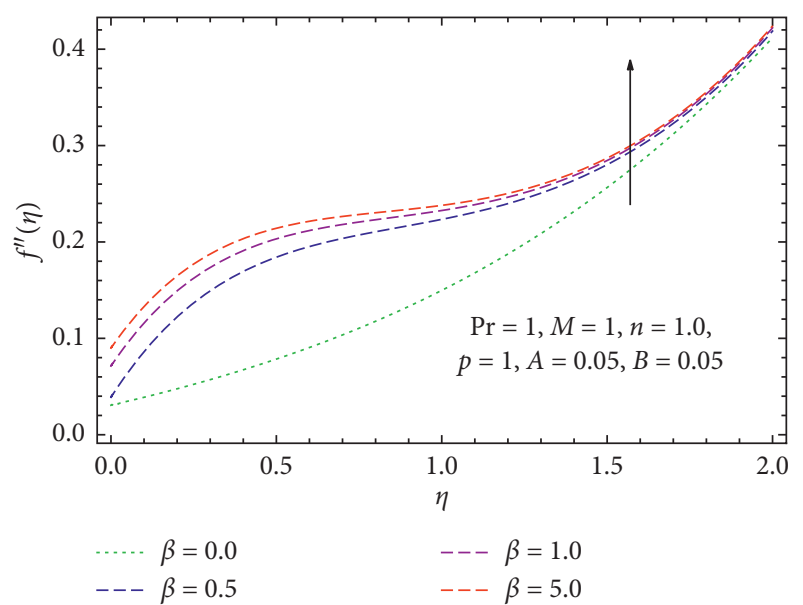

Figure 10: Skin-friction profile for different values of $\beta$.

width of the skin-friction of boundary wall layer grows wider as the values of parameter $\lambda$ increase and shrink with rising of the velocity slip flow parameter $\beta$.

Figures 11 and 12 illustrate the temperature profile for various input values of power index $n$ and some other

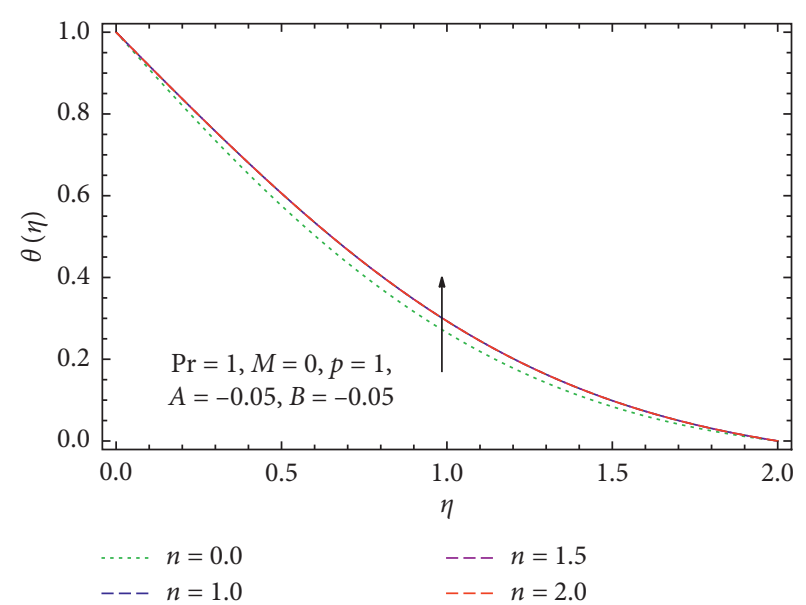

FIgURE 11: Temperature profile for different values of $n$ and different parameters.

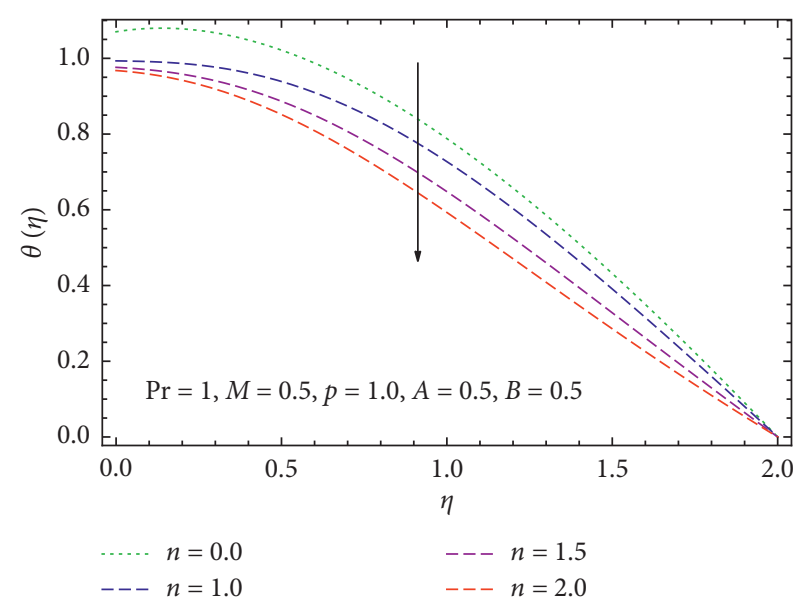

FIgURE 12: Temperature profile for different values of $n$ and different parameters.

physical variables which reveals that the thickness of the thermal-conducting boundary layer decreases with increasing the nonlinear stretching parameter $n$. Because of electromagnetic (Lorentz) force, the thickness of the heat convective boundary layer increases as the magnetic field strength increases; henceforth, Figure 13 is sketched for the temperature dependence upon the magnetic field.

Figures 14 and 15 show the temperature profiles for different inputs of Pr. These figures illustrate that, on increasing the Prandtl number Pr, the thickness of the thermal-conducting boundary layer decreases; hence, the temperature decreases.

Temperature profile for numerous values of positiondependent heat reservoir parameter $A^{*}$ and temperaturedependent heat reservoir parameter $B^{*}$, respectively, is shown in Figures 16 and 17, respectively, while the wall temperature profile is given in Figure 18 which illustrates that the temperature on the boundary layer decreases as a result of rising the power index $p$.

Figures 19 and 20 show temperature profile for the various input values of oscillating and velocity slip 


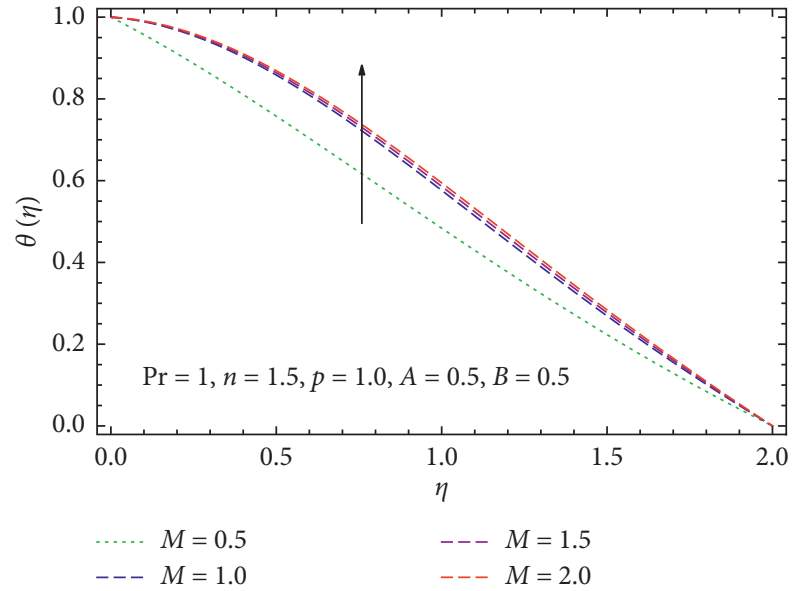

Figure 13: Temperature profile for different values of $M$.

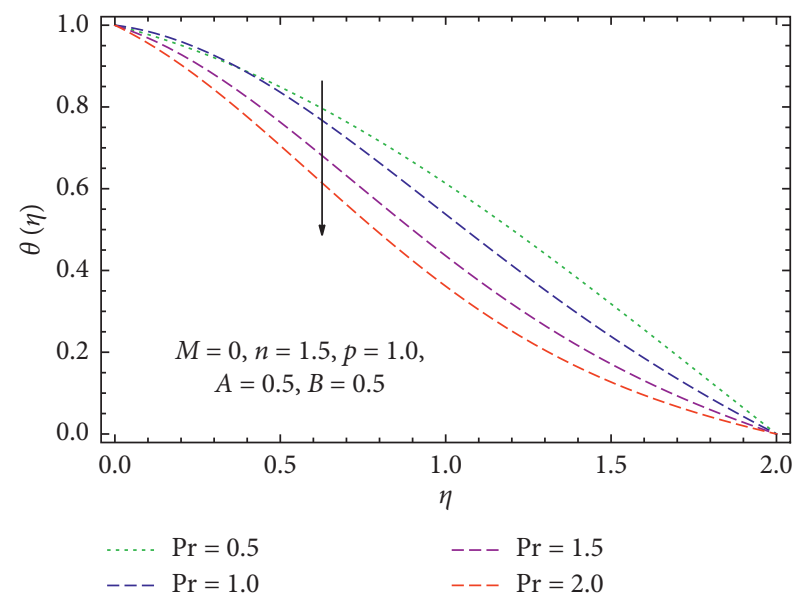

Figure 14: Temperature profile for different values of Pr.

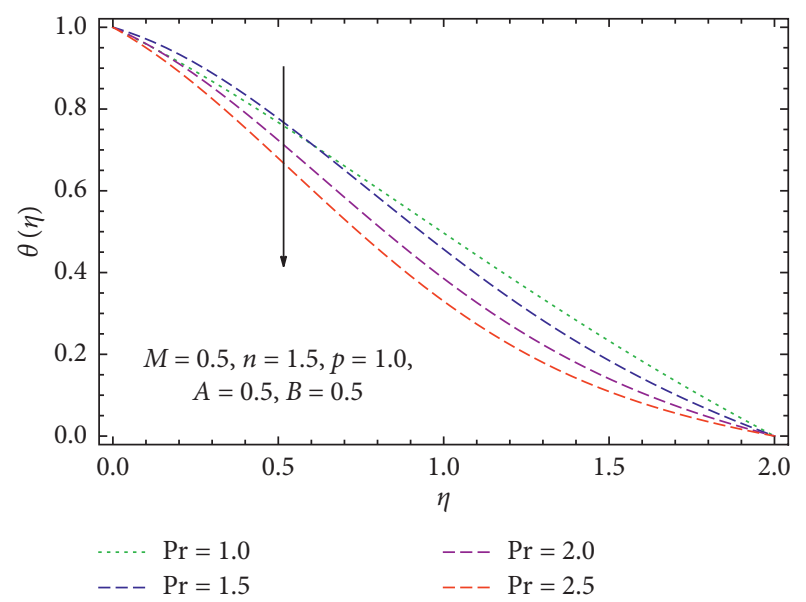

Figure 15: Temperature profile for different values of Pr.

parameters $\lambda$ and $\beta$, respectively. It is observed that, by increasing both $\lambda$ and $\beta$ parameters, the thickness of the thermal boundary layer increases.

Figures 21-23 represent the gradient of temperature sketch for different inputs of physical constrains like

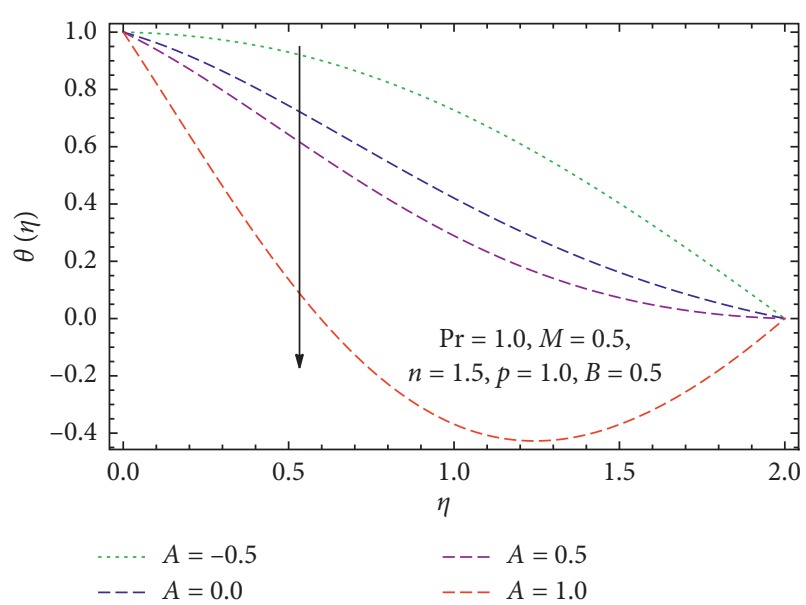

Figure 16: Temperature profile for different values of $A$.

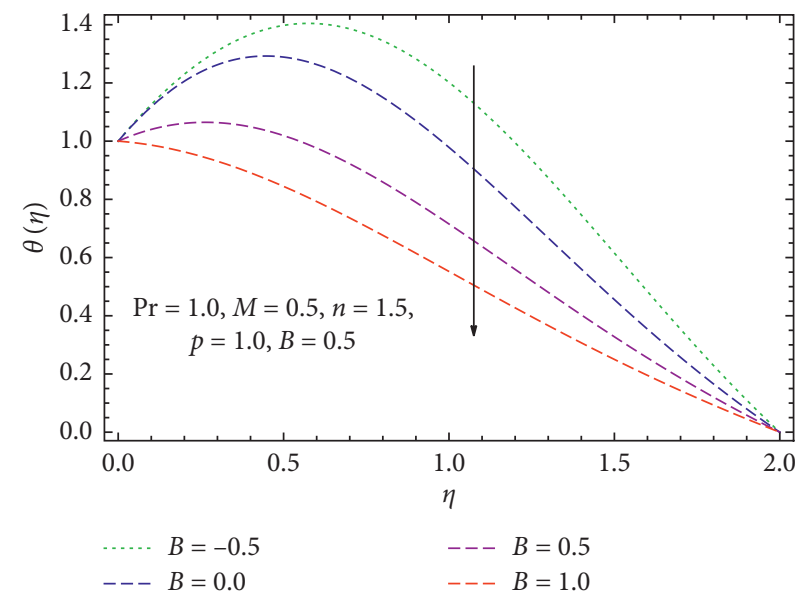

FIGURE 17: Temperature profile for different values of $B$.

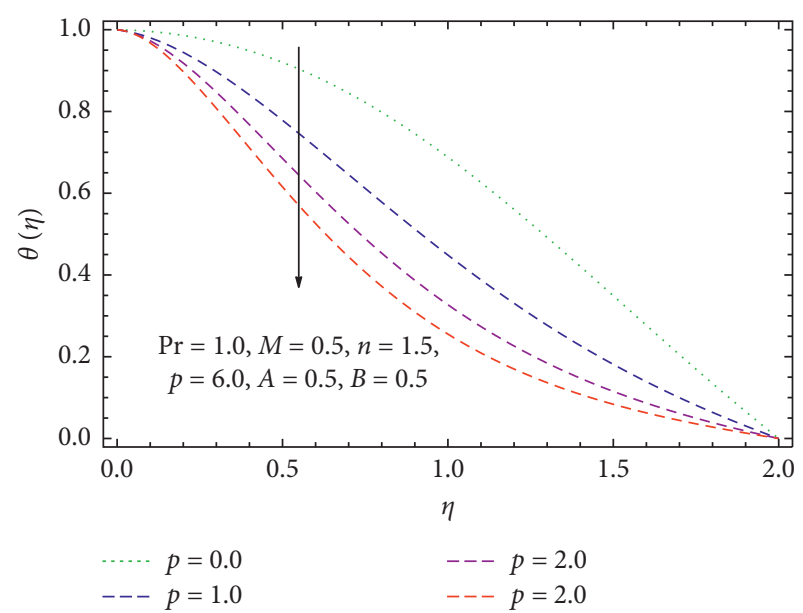

Figure 18: Temperature profile for different values of power-law index $p$.

oscillating, temperature, and velocity slip parameters, respectively. The influence of this physical parameter is observed as the temperature profile increases with the 


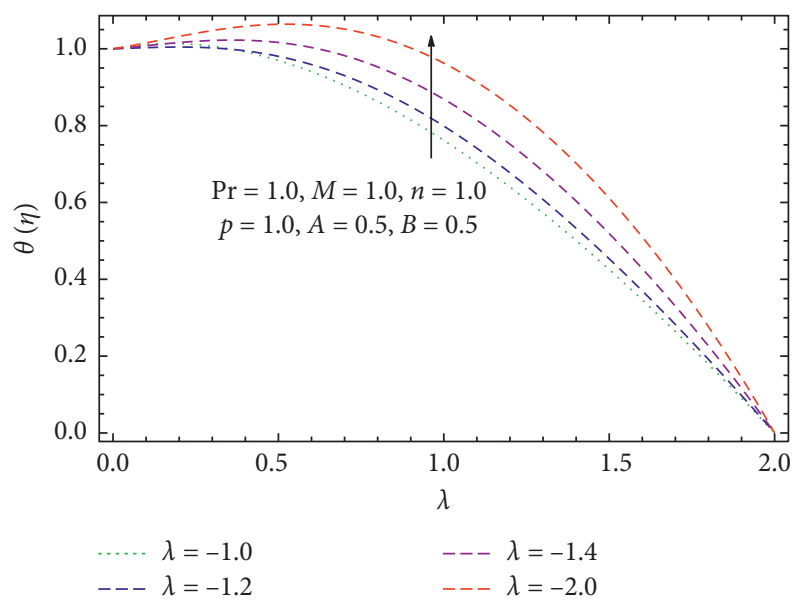

Figure 19: Temperature profile for different values of $\lambda$.

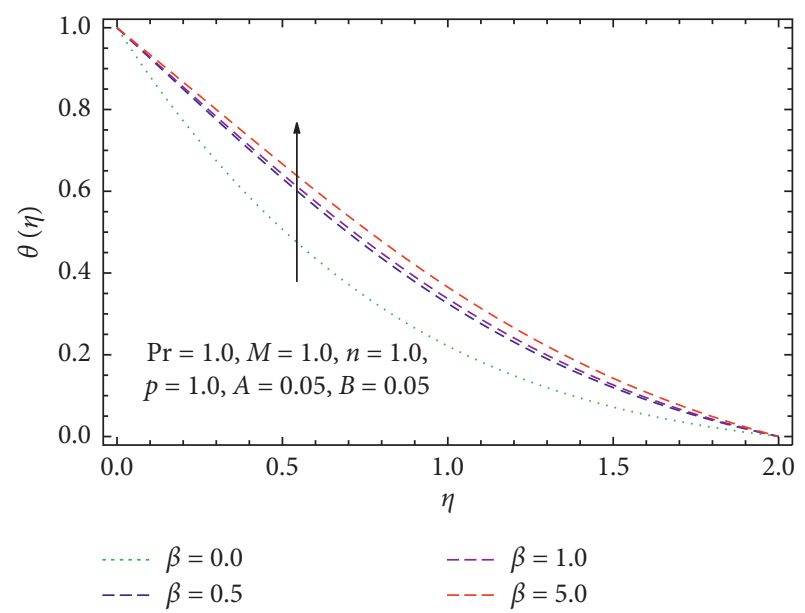

Figure 20: Temperature profile for different values of $\beta$.

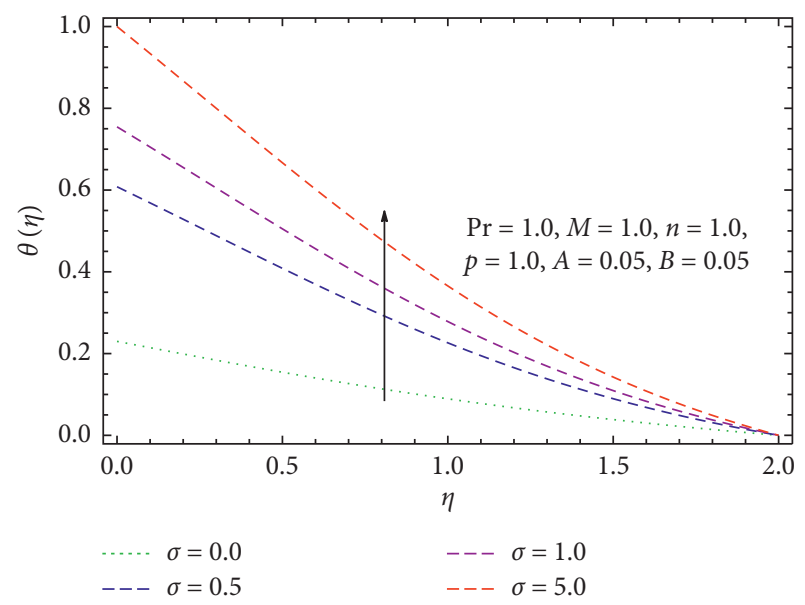

Figure 21: Temperature profile for different values of $\sigma$.

increasing value of the oscillating and velocity slip parameters, respectively, and the temperature gradient drops when the temperature slip parameter rises.

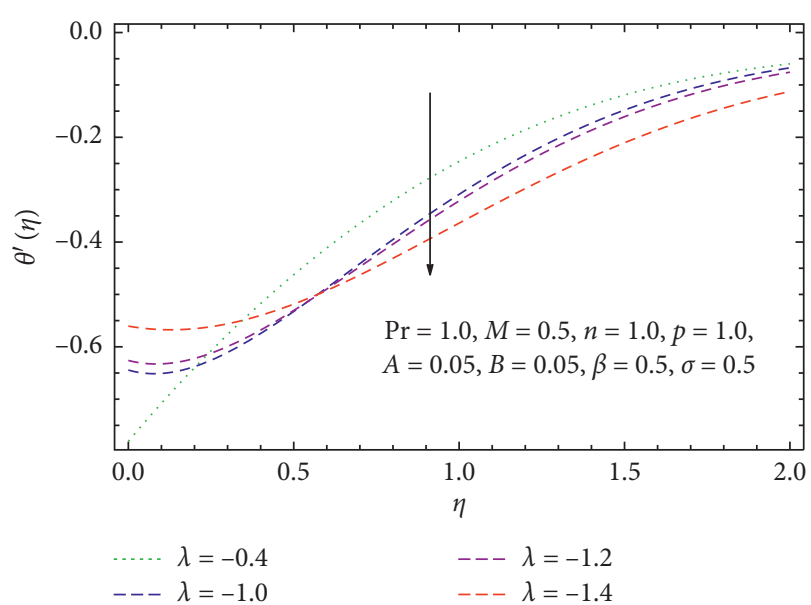

Figure 22: Temperature gradient profile for different values of $\lambda$.

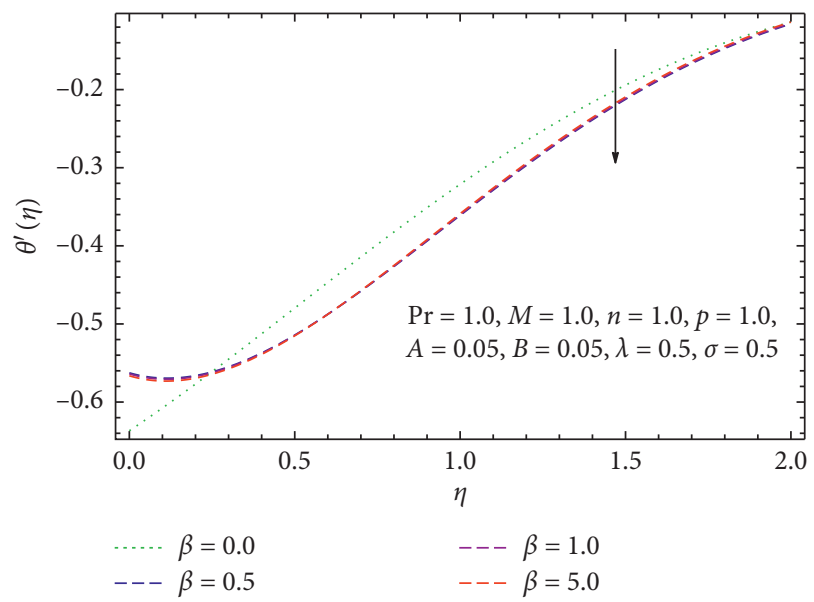

FIgURE 23: Temperature gradient profile for different values of $\beta$.

\section{Conclusions}

In this review, we have attempted to provide a glimpse of what we have studied from the stagnate point of magnetohydrodynamic flow movement and thermal convection in the presence of velocity slip, thermal slip, and nonlinear heat reservoir. We deduced some significant observations from these results given as follows:

(i) By rising the power index, the velocity parameter rises, while in existence of magnetic field, a converse result is observed.

(ii) Whether magnetic field exists or not, the velocity rises by increasing the power-law index.

(iii) If the magnetic field value is zero, the temperature rises as the power index increases while falls when magnetic field is nonzero.

(iv) As the Prandtl number increases, the temperature falls irrespective of the magnetic field.

(v) In presence of uniform magnetic field, the temperature falls down as the parameters $A^{*}, B^{*}$, and $p$ increase. Also, the temperature rises when there is a variable magnetic field. 
(vi) The oscillating parameter $\lambda$ decreases flow movement, velocity, variation of temperature in normal direction, skin friction, and heat transfer boundary layer.

(vii) The velocity slip parameter increases the thermal boundary layer and gradient of temperature but decreases the flow movement, velocity (momentum), and skin friction of the boundary interface layer.

(viii) The temperature slip parameter impacts merely on thermal interface layers which decreases the heatconvective boundary layer and so as reduces the gradient of temperature.

\section{Nomenclature}

$C_{p}: \quad$ Specific heat

Pr: Prandtl number

$T_{\infty}$ : Free stream temperature

$v$ : Velocity along $y$-axis

$A^{*}$ : Space-dependent internal heat generation

$C_{f}:$ Friction coefficient

$q_{w}$ : Wall heat flux

$T_{0}$ : Characteristic temperature

$x$ : $\quad$ Distance along the plate

$B^{*}$ : Temperature-dependent internal heat absorption

$f$ : Dimensionless stream function

$R u_{x}$ : Local Reynolds number

$T_{W}$ : Wall temperature

$y: \quad$ Distance normal to plate

$P: \quad$ Wall temperature parameter

K: $\quad$ Thermal conductivity

$n$ : Nonlinear stretching parameter

$u$ : Velocity along $x$-axis

$q^{\prime \prime \prime}:$ Nonuniform heat source/sink

$M n$ : Magnetic parameter

$N u_{x}$ : Local Nusselt number

$T$ : $\quad$ Local fluid temperature

$u_{W}$ : Velocity at wall

$B_{0}$ : Applied magnetic field

Greek Symbols

$\psi$ : Stream function

$\beta$ : Velocity slip parameter

$\lambda$ : Thermal jump parameter

$\sigma$ : Temperature slip parameter

$\alpha$ : Thermal diffusivity

$\eta$ : Similarity variable

$\mu$ : Dynamical viscosity

$\theta$ : Dimensional temperature

$v$ : Kinematic viscosity

$\rho$ : Density.

\section{Data Availability}

The data used to support the findings of this study are available from the corresponding author upon request.

\section{Conflicts of Interest}

The authors declare that they have no conflicts of interest.

\section{Acknowledgments}

The authors extend their appreciation to the Deanship of Scientific Research at Majmaah University for funding this work under Project Number (RGP-2019-3). This work was supported by the Soonchunhyang University Research Fund.

\section{References}

[1] K. K. Raju, N. Muthiyalu, and G. V. Rao, "Least square finite element solution of stagnation point flow," Complex and Fluids, vol. 4, no. 3-4, pp. 143-147, 1976.

[2] R. S. R. Gorla, "Non-Newtonian fluid at a stag-nation point in the presence of a transverse magnetic field," Mechanics Research Communications, vol. 3, no. 1, pp. 1-6, 1976.

[3] H. S. Takhar, C. D. S. Devi, and G. Nath, "MHD unsteady incompressible three-dimensional asymmetric stagnation point boundary layers," Mechanics Research Communications, vol. 14, no. 1, pp. 29-35, 1987.

[4] B. P. Besser, R. P. Rijnbeek, and H. K. Biernat, "Planar MHD stagnation point flows with velocity shear, planet," Planetary and Space Science, vol. 38, no. 3, pp. 411-418, 1990.

[5] M. Massoudi and M. Ramezan, "Heat transfer analysis of a viscoelastic fluid at a stagnation point," Mechanics Research Communications, vol. 19, no. 2, pp. 129-134, 1992.

[6] P. D. Ariel, "Three-dimensional stagnation point flow of a viscoelastic fluid," Mechanics Research Communications, vol. 21, no. 4, pp. 389-396, 1994.

[7] T. R. Mahapatra and A. S. Gupta, "Stagnation point flow towards a stretching surface," Canadian Journal of Chemical Engineering, vol. 81, pp. 258-263, 2003.

[8] M. S. Able, J. Tawade, and M. M. Nandeppanavar, "Effect of non-uniform heat source on MHD heat transfer in a liquid film over an unsteady stretching sheet," International Journal of Non-Linear Mechanics, vol. 44, no. 9, pp. 990-998, 2009.

[9] M. H. Yazdi, S. Abdullah, and I. H. Sopian, "Slip MHD flow over permeable stretching surface with chemical reaction," in Proceedings of the 17th Australian Fluid Mechanics Conference, Auckland, New Zealand, December 2010.

[10] K. L. Hsiao, "MHD stagnation point viscoelastic fluid flow and heat transfer on a thermal forming stretching sheet with viscous dissipation," The Canadian Journal of Chemical Engineering, vol. 89, no. 5, pp. 1228-1235, 2011.

[11] H. Rasheed, Z. Khan, I. Khan, D. Ching, and K. Nisar, "Numerical and analytical investigation of an unsteady thin film nanofluid flow over an angular surface," Processes, vol. 7, no. 8, p. 486, 2019.

[12] N. C. Roşca, A. V. Roşca, and I. Pop, "Stagnation point flow and heat transfer over a non-linearly moving flat plate in a parallel free stream with slip," Communications in Nonlinear Science and Numerical Simulation, vol. 19, no. 6, pp. 18221835, 2014.

[13] H. Dessie and N. Kishan, "MHD effects on heat transfer over stretching sheet embedded in porous medium with variable viscosity viscous dissipation and heat source/sink," Ain Shams Engineering Journal, vol. 5, no. 3, pp. 967-977, 2014.

[14] H. U. Rasheed, Z. Khan, S. Islam, I. Khan, J. L. G. Guirao, and W. Khan, "Investigation of two-dimensional viscoelastic fluid with nonuniform heat generation over permeable stretching 
sheet with slip condition," Complexity, vol. 2019, Article ID 3121896, 8 pages, 2019.

[15] H. S. Hassan, "Symmetry analysis for MHD viscous flow and heat transfer over a stretching sheet," Applied Mathematics, vol. 6, no. 1, pp. 78-94, 2015.

[16] M. Shen, F. Wang, and H. Chen, "MHD mixed convection slip flow near a stagnation point on a non-linearly vertical stretching sheet," Boundary Value Problems, vol. 2015, p. 78, 2015.

[17] M. N. Tufail and A. Ali, "Irreversibility analysis of MHD flow over an exponentially stretching sheet," Heat Transfer-Asian Research, vol. 44, no. 3, pp. 211-226, 2015.

[18] B. J. Gireesha, B. Mahanthesh, R. S. R. Gorla, and P. T. Manjunatha, "Thermal radiation and hall effects on boundary layer flow past a non-isothermal stretching surface embedded in porous medium with nonuniform heat source and fluid particle suspension," Heat and Mass Transfer, vol. 52, no. 4, pp. 897-911, 2016.

[19] S. Z. A. Zaidi and S. T. Mohyud-Din, "Convective heat transfer and MHD effects on two-dimensional wall jet flow of a nanofluid with passive control model," Aerospace Science and Technology, vol. 49, pp. 225-230, 2016.

[20] Z. Khan, H. U. Rasheed, I. Tlili, I. Khan, and T. Abbas, "Runge-Kutta 4th-order method analysis for viscoelastic Oldroyd 8-constant fluid used as coating material for wire with temperature dependent viscosity," Scientific Reports, vol. 8, no. 1, p. 14504, 2018.

[21] Z. Khan, W. A. Khan, H. Ur Rasheed, I. Khan, and K. S. Nisar, "Melting flow in wire coating of a third grade fluid over a die using Reynolds' and vogel's models with non-linear thermal radiation and joule heating," Materials, vol.12, no. 19, p. 3074, 2019.

[22] Z. Khan, H. Rasheed, Q. Shah, T. Abbas, and M. Ullah, "Numerical simulation of double-layer optical fiber coating using Oldroyd 8-constant fluid as a coating material," Optical Engineering, vol. 57, no. 7, Article ID 076104, 2018.

[23] Z. Khan, R. Shah, S. Islam et al., "MHD flow and heat transfer analysis in wire coating process using elastic-viscous fluid," Coatings, vol. 7, no. 1, p. 15, 2017.

[24] Z. Khan, H. U. Rasheed, M. Ullah, I. Khan, T. A. Alkanhal, and I. Tlili, "Shooting method analysis in wire coating withdrawing from a bath of Oldroyd 8-constant fluid with temperature dependent viscosity," Open Physics, vol. 16, no. 1, pp. 956-966, 2018.

[25] Z. Khan, N. Tairan, W. K. Mashwani, H. U. Rasheed, H. Shah, and W. Khan, "MHD and slip effect on two-immiscible third grade fluid on thin film flow over a vertical moving belt," Open Physics, vol. 17, no. 1, pp. 575-586, 2019.

[26] P. Kumam, Z. Shah, A. Dawar, H. U. Rasheed, and S. Islam, "Entropy generation in MHD radiative flow of CNTs Casson nanofluid in rotating channels with heat source/sink," Mathematical Problems in Engineering, vol. 2019, Article ID 9158093, 14 pages, 2019.

[27] Z. Khan, H. Ur Rasheed, M. Ullah, T. Gul, and A. Jan, "Analytical and numerical solutions of Oldroyd 8-constant fluid in double-layer optical fiber coating," Journal of Coatings Technology and Research, vol. 16, no. 1, pp. 235-248, 2018.

[28] M. M. Nandeppanavar, K. Vajravelu, M. S. Abel, and C.-O. Ng, "Heat transfer over a nonlinearly stretching sheet with non-uniform heat source and variable wall temperature," International Journal of Heat and Mass Transfer, vol. 54, no. 23-24, pp. 4960-4965, 2011.

[29] M. M. Nandeppanavar, K. Vajravelu, M. Subhas Abel, S. Ravi, and H. Jyoti, "Heat transfer in a liquid film over an unsteady stretching sheet," International Journal of Heat and Mass Transfer, vol. 55, no. 4, pp. 1316-1324, 2012.

[30] W. Ibrahim, B. Shankar, and M. M. Nandeppanavar, "Magneto hydrodynamic stagnation point flow and heat transfer due to nanofluid towards a stretching sheet," International Journal of Heat and Mass Transfer, vol. 56, no. 1-2, pp. 1-9, 2013.

[31] M. M. Nandeppanavar, K. Vajravelu, M. S. Abel, and M. N. Siddalingappa, "Second order slip flow and heat transfer over a stretching sheet with nonlinear Navier boundary condition," International Journal of Thermal Sciences, vol. 58, pp. 143-150, 2012.

[32] W. Ibrahim and R. Ul Haq, "Magneto-hydrodynamic stagnation point flow of nanofluid past a stretching sheet with convective boundary condition," Journal of the Brazilian Society of Mechanical Sciences and Engineering, vol. 38, no. 4, pp. 1155-1164, 2016.

[33] O. G. Martynenko and P. P Khramtsov, Free-Convective Heat Transfer, Springer-Verlag, Berlin, Germany, 2005.

[34] A. Bejan, Convection Heat Transfer, Wiley, New York, NY, USA, 2nd edition, 1995.

[35] Z. Khan, H. Ur Rasheed, S. O. Alharbi, I. Khan, T. Abbas, and D. Chin, "Manufacturing of double layer optical fiber coating using phan-thien-tanner fluid as coating material," Coatings, vol. 9, no. 2, p. 147, 2019.

[36] Z. Khan, H. Rasheed, T. A. Alkanhal, M. Ullah, I. Khan, and I. Tlili, "Effect of magnetic field and heat source on upperconvected-maxwell fluid in a porous channel," Open Physics, vol. 16, no. 1, pp. 917-928, 2018.

[37] Z. Khan, M. Khan, S. Islam et al., "Analysis of magnetohydrodynamics flow and heat transfer of a viscoelastic fluid through porous medium in wire coating analysis," Mathematics, vol. 5, no. 2, p. 27, 2017. 